\title{
Axial coupling constant of the nucleon for two flavors of dynamical quarks in finite and infinite volume
}

\author{
A. Ali Khan, ${ }^{1}$ M. Göckeler, ${ }^{1}$ Ph. Hägler, ${ }^{2}$ T. R. Hemmert, ${ }^{2}$ R. Horsley, ${ }^{3}$ D. Pleiter, ${ }^{4}$ P. E. L. Rakow, ${ }^{5}$ A. Schäfer, ${ }^{1}$ \\ G. Schierholz, ${ }^{4,6}$ T. Wollenweber, ${ }^{2}$ and J. M. Zanotti ${ }^{3}$
}

(QCDSF Collaboration)

\author{
${ }^{1}$ Institut für Theoretische Physik, Universität Regensburg, 93040 Regensburg, Germany \\ ${ }^{2}$ Physik-Department, Theoretische Physik, Technische Universität München, 85747 Garching, Germany \\ ${ }^{3}$ School of Physics, University of Edinburgh, Edinburgh EH9 3JZ, United Kingdom \\ ${ }^{4} J o h n$ von Neumann-Institut für Computing NIC, Deutsches Elektronen-Synchrotron DESY, 15738 Zeuthen, Germany \\ ${ }^{5}$ Theoretical Physics Division, Department of Mathematical Sciences, University of Liverpool, Liverpool L69 3BX, United Kingdom \\ ${ }^{6}$ Deutsches Elektronen-Synchrotron DESY, 22603 Hamburg, Germany \\ (Received 18 April 2006; published 27 November 2006)
}

\begin{abstract}
We present data for the axial coupling constant $g_{A}$ of the nucleon obtained in lattice QCD with two degenerate flavors of dynamical nonperturbatively improved Wilson quarks. The renormalization is also performed nonperturbatively. For the analysis we give a chiral extrapolation formula for $g_{A}$ based on the small scale expansion scheme of chiral effective field theory for two degenerate quark flavors. Applying this formalism in a finite volume, we derive a formula that allows us to extrapolate our data simultaneously to the infinite volume and to the chiral limit. Using the additional lattice data in finite volume, we are able to determine the axial coupling of the nucleon in the chiral limit without imposing the known value at the physical point.
\end{abstract}

DOI: 10.1103/PhysRevD.74.094508

PACS numbers: 11.15.Ha, 12.38.Gc, 12.39.Fe

\section{INTRODUCTION}

The axial coupling constant $g_{A}$ of the nucleon has been studied theoretically as well as experimentally for many years. It can be defined as the value of the axial form factor of the nucleon at vanishing 4-momentum transfer. More explicitly, one considers the isovector axial current $A_{\mu}^{u-d}=$ $\bar{u} \gamma_{\mu} \gamma_{5} u-\bar{d} \gamma_{\mu} \gamma_{5} d$, where $u$ and $d$ denote the up and down quark fields, respectively. We work in the limit of exact isospin invariance, i.e. $u$ and $d$ quarks are assumed to be degenerate in mass. The proton matrix element of this current has the form factor decomposition

$$
\begin{aligned}
\left\langle p^{\prime}, s^{\prime}\left|A_{\mu}^{u-d}\right| p, s\right\rangle= & \bar{u}\left(p^{\prime}, s^{\prime}\right)\left[\gamma_{\mu} \gamma_{5} G_{A}\left(q^{2}\right)\right. \\
& \left.+\gamma_{5} \frac{q_{\mu}}{2 m_{N}} G_{P}\left(q^{2}\right)\right] u(p, s) .
\end{aligned}
$$

Here $q=p^{\prime}-p$ denotes the 4-momentum transfer and $u(p, s)$ is the proton spinor for momentum $p$ and spin vector $s$. The states are normalized according to $\left\langle p^{\prime}, s^{\prime} \mid p, s\right\rangle=(2 \pi)^{3} 2 p^{0} \delta\left(\vec{p}-\vec{p}^{\prime}\right) \delta_{s s^{\prime}}$, we take $s^{2}=$ $-m_{N}^{2}$, and $m_{N}$ is the nucleon mass. So $g_{A}=G_{A}(0)$ is determined by the forward matrix element

$$
\left\langle p, s\left|A_{\mu}^{u-d}\right| p, s\right\rangle=2 g_{A} s_{\mu} .
$$

In parton model language, the forward matrix elements of the axial current are related to the fraction of the nucleon spin carried by the quarks. Denoting by $\Delta u(\Delta d)$ the contribution of the $u(d)$ quarks, one has

$$
\left\langle p, s\left|\bar{u} \gamma_{\mu} \gamma_{5} u\right| p, s\right\rangle=2 \Delta u s_{\mu}
$$

and similarly for the $d$ quarks. Thus, we can write $g_{A}=$ $\Delta u-\Delta d$.

In this paper we report on new results for $g_{A}$ obtained by means of Monte Carlo simulations of lattice QCD with two dynamical quark flavors. While Eq. (2) lends itself immediately to an evaluation on the lattice, it is not yet possible to perform the simulations at physical quark masses. Moreover, the size of the lattice is necessarily finite and in practice not very large. Thus, apart from the unavoidable continuum extrapolation, we have to cope with the thermodynamic limit and the extrapolation to small (physical) quark masses, the so-called chiral extrapolation. Guidance for these extrapolations is provided by chiral effective field theory (ChEFT).

In its standard form, ChEFT describes low-energy QCD by means of an effective field theory based on pion, nucleon, ... fields taking into account the constraints imposed by (spontaneously broken) chiral symmetry. As long as one stays in the $p$-regime of ChEFT (with appropriate boundary conditions), the Lagrangian does not depend on the volume. So besides the quark-mass dependence, the very same Lagrangian governs also the volume dependence, and finite size effects can be calculated by evaluating the theory in a finite (spatial) volume. Thus, the finite volume does not introduce any new parameters and the study of the finite size effects yields an additional handle on the coupling constants of ChEFT. 
There are several ways to treat baryons in ChEFT. Here we apply the (nonrelativistic) small scale expansion (SSE) [1], which uses explicit pion, nucleon, and $\Delta(1232)$ degrees of freedom, and extend the previous calculations of the quark-mass dependence of $g_{A}[2,3]$ in this scheme to finite volume. The dependence on the lattice spacing $a$ could be included [4] (for a review see Ref. [5]), but we shall not consider this possibility here.

A somewhat more phenomenological approach to the chiral extrapolation of $g_{A}$ (and other nucleon matrix elements) has been developed in Ref. [6]. The volume dependence of such matrix elements has also been studied by several methods [7-9] and we shall compare our procedure in some detail with that of Beane and Savage [7].

Preliminary results of our investigation have been presented in Refs. [10,11].

\section{THE SIMULATIONS}

The QCDSF and UKQCD collaborations have generated ensembles of gauge field configurations using $N_{f}=2$ nonperturbatively $\mathcal{O}(a)$ improved Wilson quarks and Wilson's plaquette action for the gauge fields. The simulation parameters are listed in Table I along with some auxiliary results needed later on. Note that we have two groups of three ensembles each which differ only in the volume (simulations 9, 10, 11 and 12, 13, 14). In this paper we shall not consider any partially quenched results, hence we set $\kappa=\kappa_{\text {sea }}$.

When we want to compare (fit) our results with formulas from ChEFT, we need all numbers in physical units, i.e. we have to fix the scale. This is usually done with the help of the force scale $r_{0}$ [12] derived from the heavy-quark po- tential. From phenomenology we know that $r_{0} \approx 0.5 \mathrm{fm}$ in the real world. Unfortunately, it is not easy to determine this value precisely because it is related to measurable quantities only through the use of potential models.

Yet this is not the only problem with setting the scale. In the analysis of quenched simulations, it is common practice to identify $r_{0}$ as extracted from the Monte Carlo data with the physical $r_{0}$. However, the latter refers to a world where the sea quarks have their physical masses while in the quenched simulations they are infinitely heavy. Still, for many quantities quenched calculations lead to results that agree surprisingly well with experiment.

In simulations with dynamical quarks additional difficulties arise. In this case $r_{0} / a$ depends not only on $\beta$ as in the quenched approximation, but also on $\kappa$. Then one can either identify the value for $r_{0}$ obtained at the given $(\beta, \kappa)$ combination with the physical $r_{0}$ or one can attempt an extrapolation of $r_{0} / a$ to the chiral limit, e.g. at fixed $\beta$, and use the extrapolated value to set the scale. The first procedure is analogous to the standard approach in the quenched case and ensures a continuous connection with the quenched results as the quark mass tends to infinity. The second procedure, on the other hand, has the virtue of leading to a mass-independent lattice spacing $a$. It is however to be noted that, strictly speaking, the chiral extrapolation should be performed at fixed $\tilde{g}_{0}^{2}=\left(1+b_{g} a m\right) g_{0}^{2}$ (see Ref. [13]) and not at fixed $\beta$, although the difference seems to be rather small in practice.

We have determined $r_{0}$ in physical units from the nucleon mass. Of course, this can only be done after a chiral extrapolation to the physical pion mass. Encouraged by our comparison of nucleon mass data in different volumes with chiral perturbation theory [14], we have used the extrapo-

TABLE I. Simulation parameters together with results for the force scale $r_{0}$ and the pion mass $m_{\pi}$ in lattice units. Also the value of the plaquette $u_{0}^{4}$ is given.

\begin{tabular}{lccccccc}
\hline \hline & Collaboration & $\beta$ & $\kappa_{\text {sea }}$ & Volume & $r_{0} / a$ & $a m_{\pi}$ & $u_{0}^{4}$ \\
\hline 1 & QCDSF & 5.20 & 0.1342 & $16^{3} \times 32$ & $4.077(70)$ & $0.5847(12)$ & $0.528994(58)$ \\
2 & UKQCD & 5.20 & 0.1350 & $16^{3} \times 32$ & $4.754(45)$ & $0.4148(13)$ & $0.533670(40)$ \\
3 & UKQCD & 5.20 & 0.1355 & $16^{3} \times 32$ & $5.041(53)$ & $0.2907(15)$ & $0.536250(30)$ \\
4 & QCDSF & 5.25 & 0.1346 & $16^{3} \times 32$ & $4.737(50)$ & $0.4932(10)$ & $0.538770(41)$ \\
5 & UKQCD & 5.25 & 0.1352 & $16^{3} \times 32$ & $5.138(55)$ & $0.3821(13)$ & $0.541150(30)$ \\
6 & QCDSF & 5.25 & 0.13575 & $24^{3} \times 48$ & $5.532(40)$ & $0.2556(5)$ & $0.543135(15)$ \\
7 & UKQCD & 5.29 & 0.1340 & $16^{3} \times 32$ & $4.813(82)$ & $0.5767(11)$ & $0.542400(50)$ \\
8 & QCDSF & 5.29 & 0.1350 & $16^{3} \times 32$ & $5.227(75)$ & $0.4206(9)$ & $0.545520(29)$ \\
9 & QCDSF & 5.29 & 0.1355 & $12^{3} \times 32$ & $\ldots$ & $\ldots$ & $\ldots$ \\
10 & QCDSF & 5.29 & 0.1355 & $16^{3} \times 32$ & $\ldots$ & $\ldots$ & $\ldots$ \\
11 & QCDSF & 5.29 & 0.1355 & $24^{3} \times 48$ & $5.566(64)$ & $0.3269(7)$ & $0.547094(23)$ \\
12 & QCDSF & 5.29 & 0.1359 & $12^{3} \times 32$ & $\ldots$ & $\ldots$ & $\ldots$ \\
13 & QCDSF & 5.29 & 0.1359 & $16^{3} \times 32$ & $\ldots$ & $\ldots$ & $\ldots$ \\
14 & QCDSF & 5.29 & 0.1359 & $24^{3} \times 48$ & $5.88(10)$ & $0.2392(9)$ & $0.548286(57)$ \\
15 & QCDSF & 5.40 & 0.1350 & $24^{3} \times 48$ & $6.092(67)$ & $0.4030(4)$ & $0.559000(19)$ \\
16 & QCDSF & 5.40 & 0.1356 & $24^{3} \times 48$ & $6.381(53)$ & $0.3123(7)$ & $0.560246(10)$ \\
17 & QCDSF & 5.40 & 0.1361 & $24^{3} \times 48$ & $6.714(64)$ & $0.2208(7)$ & $0.561281(8)$ \\
\hline \hline
\end{tabular}


lation procedure described in [14] as Fit 1 to recent nucleon masses obtained by the CP-PACS and JLQCD collaborations along with updated masses from the QCDSFUKQCD collaboration. Varying the assumed physical value of $r_{0}$, one can make the fit curve pass through the physical point, which happens for $r_{0}=0.467 \mathrm{fm}$. This number is consistent with a recent lattice calculation of $F_{\pi}$ [15], giving $r_{0}=0.475(25) \mathrm{fm}$. A similar result for $r_{0}$ was also quoted in [16] taking as input level splittings in the $Y$ spectrum. In the following, we shall use $r_{0}=$ $0.467 \mathrm{fm}$, but for comparison we shall also consider $r_{0}=$ $0.5 \mathrm{fm}$.

We compute $g_{A}$ from forward proton matrix elements (2) of the flavor-nonsinglet axial-vector current at $\vec{p}=\overrightarrow{0}$. The required bare matrix elements are extracted from ratios of 3-point functions over 2-point functions in the standard fashion. Our results for $g_{A}^{\text {bare }}$ are collected in Table II. Compared to the computation of hadron masses, additional difficulties may arise in the calculation of nucleon matrix elements such as $g_{A}$ : In general there are quark-line disconnected contributions, which are hard to evaluate; the operators must be improved and renormalized, etc. Fortunately, in the limit of exact isospin invariance, which is taken in our simulations, all disconnected contributions cancel in $g_{A}$, because it is a flavor-nonsinglet quantity. So we do not have to worry about any disconnected contributions.

The axial-vector current is $\mathcal{O}(a)$ improved by adding the derivative of the pseudoscalar density with the appropriate coefficient:

$$
A_{\mu}^{\mathrm{imp}}(x)=\bar{q}(x) \gamma_{\mu} \gamma_{5} q(x)+a c_{A}^{\mathrm{imp}} \partial_{\mu} \bar{q}(x) \gamma_{5} q(x) .
$$

Hence, the improvement term does not contribute in forward matrix elements such as (2). We have denoted the improvement coefficient (usually called $c_{A}$ ) by $c_{A}^{\text {imp in }}$ order to avoid confusion with a coupling constant appearing later.

The renormalized improved axial-vector current can be written as

$$
A_{\mu}=Z_{A}\left(1+b_{A} a m\right) A_{\mu}^{\mathrm{imp}}
$$

with the bare quark mass $m=\left(1 / \kappa-1 / \kappa_{c}\right) /(2 a)$. The values of $\kappa_{c}$ used here can be found in Table III. The coefficient $b_{A}$ is required to maintain $O(a)$ improvement also for nonvanishing quark mass. We are not aware of a nonperturbative evaluation of this coefficient for our action. Hence, we have to resort to perturbation theory. A one-loop calculation yields [19]

$$
b_{A}=1+b_{A}^{(1)} g_{0}^{2}+\mathcal{O}\left(g_{0}^{4}\right), \quad b_{A}^{(1)}=0.11414(4) C_{F},
$$

where $C_{F}=4 / 3$ in QCD and $g_{0}$ denotes the bare coupling with $\beta=6 / g_{0}^{2}$. We shall use the tadpole improved version of this result, i.e. we take [20]

$$
b_{A}^{\mathrm{TI}}=u_{0}^{-1}\left(1+\left(b_{A}^{(1)}-\frac{1}{12}\right) \frac{g_{0}^{2}}{u_{0}^{4}}\right) .
$$

Here $u_{0}$ is the fourth root of the expectation value of the plaquette with the perturbative expansion

$$
u_{0}=1-\frac{1}{12} g_{0}^{2}+\mathcal{O}\left(g_{0}^{4}\right)
$$

While the coefficient $b_{A}$ will be computed in tadpole improved one-loop perturbation theory, we calculate the renormalization factor $Z_{A}$ nonperturbatively by means of the Rome-Southampton method $[21,22]$. Thus, $Z_{A}$ is first obtained in the so-called RI'-MOM scheme. Using continuum perturbation [23] theory, we switch to the $\overline{\mathrm{MS}}$ scheme. For sufficiently large renormalization scales $\mu, Z_{A}$ should

TABLE II. Bare and renormalized results for $g_{A}$. The values for $L$ and $m_{\pi}$ in physical units have been calculated using $r_{0}=0.467 \mathrm{fm}$ together with $r_{0} / a$ at the respective quark masses.

\begin{tabular}{lcccclcc}
\hline \hline & $\beta$ & $\kappa_{\text {sea }}$ & Volume & $L[\mathrm{fm}]$ & $m_{\pi}[\mathrm{GeV}]$ & $g_{A}^{\text {bare }}$ & $g_{A}$ \\
\hline 1 & 5.20 & 0.1342 & $16^{3} \times 32$ & 1.84 & $1.007(17)$ & $1.452(17)$ & $1.185(16)$ \\
2 & 5.20 & 0.1350 & $16^{3} \times 32$ & 1.57 & $0.8332(83)$ & $1.514(29)$ & $1.201(24)$ \\
3 & 5.20 & 0.1355 & $16^{3} \times 32$ & 1.48 & $0.6192(73)$ & $1.396(36)$ & $1.088(29)$ \\
4 & 5.25 & 0.1346 & $16^{3} \times 32$ & 1.58 & $0.987(11)$ & $1.442(13)$ & $1.176(12)$ \\
5 & 5.25 & 0.1352 & $16^{3} \times 32$ & 1.45 & $0.8295(93)$ & $1.438(20)$ & $1.148(17)$ \\
6 & 5.25 & 0.13575 & $24^{3} \times 48$ & 2.03 & $0.5975(45)$ & $1.456(10)$ & $1.1398(98)$ \\
7 & 5.29 & 0.1340 & $16^{3} \times 32$ & 1.55 & $1.173(20)$ & $1.437(12)$ & $1.207(12)$ \\
8 & 5.29 & 0.1350 & $16^{3} \times 32$ & 1.43 & $0.929(13)$ & $1.409(12)$ & $1.143(11)$ \\
9 & 5.29 & 0.1355 & $12^{3} \times 32$ & 1.01 & $\ldots$ & $1.181(60)$ & $0.942(48)$ \\
10 & 5.29 & 0.1355 & $16^{3} \times 32$ & 1.34 & $\ldots$ & $1.364(25)$ & $1.087(21)$ \\
11 & 5.29 & 0.1355 & $24^{3} \times 48$ & 2.01 & $0.7688(90)$ & $1.459(11)$ & $1.163(11)$ \\
12 & 5.29 & 0.1359 & $12^{3} \times 32$ & 0.95 & $\ldots$ & $0.97(10)$ & $0.763(79)$ \\
13 & 5.29 & 0.1359 & $16^{3} \times 32$ & 1.27 & $\ldots$ & $1.253(45)$ & $0.985(36)$ \\
14 & 5.29 & 0.1359 & $24^{3} \times 48$ & 1.91 & $0.594(10)$ & $1.413(22)$ & $1.111(18)$ \\
15 & 5.40 & 0.1350 & $24^{3} \times 48$ & 1.84 & $1.037(11)$ & $1.4737(74)$ & $1.2234(88)$ \\
16 & 5.40 & 0.1356 & $24^{3} \times 48$ & 1.76 & $0.8420(72)$ & $1.451(11)$ & $1.180(11)$ \\
17 & 5.40 & 0.1361 & $24^{3} \times 48$ & 1.67 & $0.6264(63)$ & $1.410(20)$ & $1.127(17)$ \\
\hline \hline
\end{tabular}


TABLE III. Values of $Z_{A}$ from this work and from the ALPHA collaboration. The latter numbers were obtained from Eq. (3.6) in Ref. [17], where an error decreasing from 0.01 at $\beta=$ 5.2 to 0.005 at $\beta=5.7$ is ascribed to them. In addition we give $\kappa_{c}$ and the values of $r_{0} / a$ extrapolated to the chiral limit (from [18]).

\begin{tabular}{lllll}
\hline \hline$\beta$ & \multicolumn{1}{c}{5.20} & \multicolumn{1}{c}{5.25} & \multicolumn{1}{c}{5.29} & \multicolumn{1}{c}{5.40} \\
\hline$Z_{A}$ (this work) & $0.765(5)$ & $0.769(4)$ & $0.772(4)$ & $0.783(4)$ \\
$Z_{A}$ (ALPHA) & 0.719 & 0.734 & 0.745 & 0.767 \\
$\kappa_{c}$ & $0.136008(15)$ & $0.136250(7)$ & $0.136410(9)$ & $0.136690(22)$ \\
$\left(r_{0} / a\right)_{\text {ch.l. }}$ & $5.455(96)$ & $5.885(79)$ & $6.254(99)$ & $7.39(26)$ \\
\hline \hline
\end{tabular}

then be independent of $\mu$. However, unless $\mu \ll 1 / a$ lattice artifacts may spoil this behavior. Since our scales do not always satisfy this criterion, we try to correct for this mismatch by subtracting the lattice artifacts perturbatively with the help of boosted one-loop lattice perturbation theory. As Fig. 1 shows, some lattice artifacts still remain, but we can nevertheless estimate $Z_{A}$. As our central value we take the result obtained at $\mu^{2}=5 \mathrm{GeV}^{2}$ while the difference to the result at $\mu^{2}=10 \mathrm{GeV}^{2}$ gives us an estimate of the (systematic) error. Adding the statistical error in quadrature, we arrive at the numbers given in Table III, where we also compare our results with a recent determination of $Z_{A}$ by the ALPHA collaboration [17]. While the numbers differ significantly at the lower $\beta$ values, there is a tendency towards agreement as $\beta$ grows. This suggests that the differences are mainly due to lattice artifacts and will not influence the continuum limit of renormalized quantities.

Our results for $g_{A}$ renormalized in the way just described are given in the last column of Table II and are plotted in Fig. 2. Note that the error on $g_{A}$ is dominated by the statistical error of the bare values while the uncertainty of $Z_{A}$ contributes only comparatively little. We have taken $m_{\pi}$ from the largest available lattice at each $(\beta, \kappa)$ combination. The scale has been set by means of the force

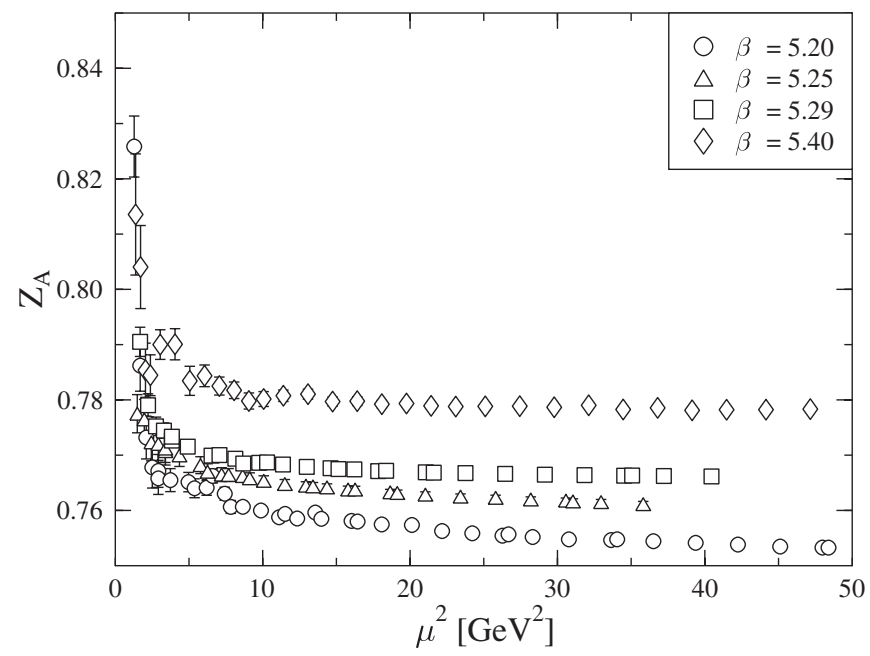

FIG. 1. The renormalization constant $Z_{A}$ plotted versus the square of the renormalization scale $\mu$. parameter $r_{0}$ with $r_{0}=0.467 \mathrm{fm}$, and $r_{0} / a$ has been taken at the given quark mass. Obviously there are considerable finite size effects. A qualitatively similar volume dependence has already been observed in quenched simulations [24]. Note that the "large volume" results for $g_{A}$ obtained at our smallest quark masses $\left(m_{\pi} \approx 600 \mathrm{MeV}\right)$ for all four $\beta$ values lie very close together indicating that discretization effects are small.

Our results may be compared to other evaluations of $g_{A}$ in dynamical simulations [25-27]. They all differ in the lattice actions employed: In Ref. [25] unimproved Wilson fermions are used, the RBCK collaboration [26] works with domain wall fermions, and the most recent LHPC investigation [27] chooses a hybrid approach with domain wall valence quarks on improved staggered sea quarks. All these studies obtain a rather weak quark-mass dependence of $g_{A}$ in agreement with our findings. However, most of their values lie somewhat above ours. At the moment, the reason for these discrepancies is not yet clear. One possibility is the different treatment of the renormalization. The perturbative renormalization employed in Ref. [25] entails a relatively large uncertainty because it relies on a one-loop

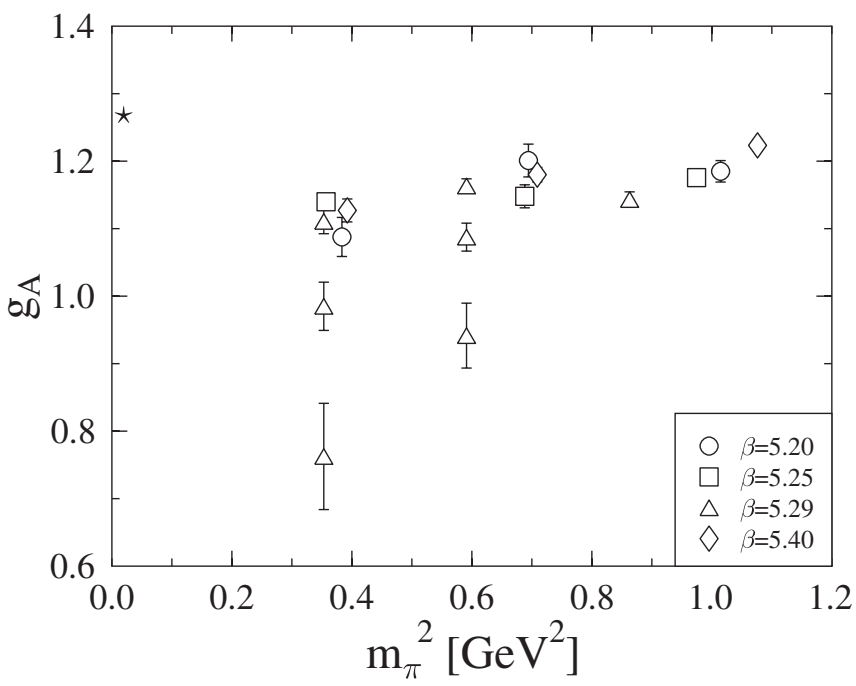

FIG. 2. Results for $g_{A}$. For $(\beta, \kappa)=(5.29,0.1355)$ and $(5.29$, $0.1359)$ we show the results obtained on three different spatial volumes. The smallest box size $L$, leading to the smallest $g_{A}$, is about $1.0 \mathrm{fm}$, while the middle size is approximately $1.3 \mathrm{fm}$. All other volumes are larger. The star represents the physical point. 
calculation only and in general lattice perturbation theory does not converge very rapidly. References $[26,27]$ exploit the fact that in the framework of domain wall fermions there are five-dimensional (partially) conserved axial and vector currents. One should however keep in mind that they are exactly conserved only in the limit of an infinite fifth dimension, while the actual simulations are necessarily performed with this extension kept finite.

Note that, using the $Z_{A}$ results of the ALPHA collaboration, we would have obtained even smaller numbers. As indicated above, this difference may be expected to disappear in the continuum limit. However, from the comparison of our results at different values of $\beta$ we have the impression that lattice artifacts are rather small in the data renormalized with our $Z_{A}$ values (see Fig. 2). This is less so when the $Z_{A}$ of the ALPHA collaboration is used. In any case, there is still some work to be done before we can be sure that all systematic uncertainties are under control.

\section{CHIRAL EFFECTIVE FIELD THEORY (INFINITE VOLUME)}

At low energies, one can evaluate the matrix element (1) of the isovector axial current of the nucleon within a lowenergy effective theory of QCD utilizing the methods of ChEFT. In the forward limit [Eq. (2)], one then obtains the axial coupling constant $g_{A}$. In the SSE formalism the results appear as expansions in powers of a small parameter $\epsilon$, which collectively denotes small pion four-momenta, the pion mass, baryon three-momenta, and the nucleon- $\Delta$ mass splitting in the chiral limit [1]. At $\mathcal{O}\left(\epsilon^{3}\right)$, one has to evaluate 8 diagrams [2], which are displayed in Fig. 3, involving nucleon, pion, and $\Delta(1232)$ degrees of freedom. When ChEFT calculations of nucleon properties using just pions and nucleons as the active degrees of freedom are extended to ChEFT calculations of the same quantities also employing explicit $\Delta(1232)$ degrees of freedom, one obtains renormalizations of the chiral limit couplings of the nucleon by polynomial terms $\sim\left(\Delta_{0}\right)^{n}$, where $\Delta_{0}$ denotes the (finite) $N-\Delta$ mass splitting in the chiral limit. This would naively imply that the chiral limit properties of a nucleon differ between ChEFT schemes with and without resonance degrees of freedom. In the SSE formalism such an unphysical scenario is automatically avoided because $\Delta_{0}$ (by construction) is treated as a quantity of $\mathcal{O}(\epsilon)$ leading to extra terms in the nucleon Lagrangian which cancel the contributions causing this behavior. We refer to Ref. [28] for a detailed example of such a decoupling construction.

Implementing this decoupling of the $\Delta$ resonance near the chiral limit leads to the result [3]

$$
\begin{aligned}
g_{A}^{\mathrm{SSE}}(\infty)= & g_{A}^{0}-\frac{\left(g_{A}^{0}\right)^{3} m_{\pi}^{2}}{16 \pi^{2} F_{\pi}^{2}}+4\left\{C^{\mathrm{SSE}}(\lambda)+\frac{c_{A}^{2}}{4 \pi^{2} F_{\pi}^{2}}\left[\frac{155}{972} g_{1}-\frac{17}{36} g_{A}^{0}\right]+\gamma^{\mathrm{SSE}} \ln \frac{m_{\pi}}{\lambda}\right\} m_{\pi}^{2}+\frac{4 c_{A}^{2} g_{A}^{0}}{27 \pi F_{\pi}^{2} \Delta_{0}} m_{\pi}^{3} \\
& +\frac{8}{27 \pi^{2} F_{\pi}^{2}} c_{A}^{2} g_{A}^{0} m_{\pi}^{2} \sqrt{1-\frac{m_{\pi}^{2}}{\Delta_{0}^{2}}} \ln R+\frac{c_{A}^{2} \Delta_{0}^{2}}{81 \pi^{2} F_{\pi}^{2}}\left(25 g_{1}-57 g_{A}^{0}\right)\left\{\ln \left[\frac{2 \Delta_{0}}{m_{\pi}}\right]-\sqrt{1-\frac{m_{\pi}^{2}}{\Delta_{0}^{2}}} \ln R\right\}+\mathcal{O}\left(\epsilon^{4}\right)
\end{aligned}
$$

with

$$
\begin{aligned}
\gamma^{\mathrm{SSE}} & =\frac{1}{16 \pi^{2} F_{\pi}^{2}}\left[\frac{50}{81} c_{A}^{2} g_{1}-\frac{1}{2} g_{A}^{0}-\frac{2}{9} c_{A}^{2} g_{A}^{0}-\left(g_{A}^{0}\right)^{3}\right], \\
R & =\frac{\Delta_{0}}{m_{\pi}}+\sqrt{\frac{\Delta_{0}^{2}}{m_{\pi}^{2}}-1 .}
\end{aligned}
$$

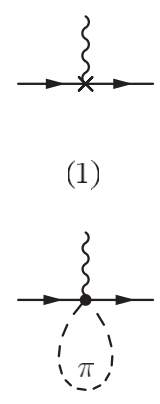

(5)

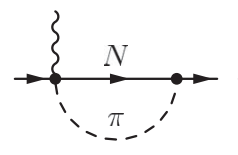

(2)

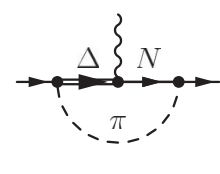

(6)

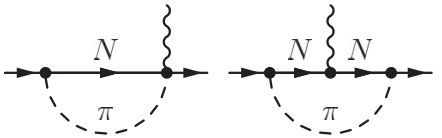

(3)

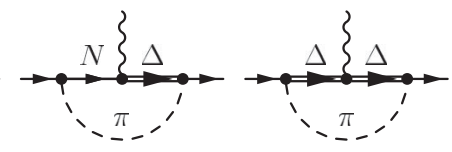

(7)
In the SSE counterterm combination [3]

$$
C^{\mathrm{SSE}}(\lambda)=B_{9}^{r}(\lambda)^{\mathrm{SSE}}-2 g_{A}^{0} B_{20}^{r}(\lambda)^{\mathrm{SSE}},
$$

$\lambda$ denotes the scale utilized in dimensional regularization, which can be freely chosen as the ChEFT results of course do not depend on the employed regularization scheme or scale. In order to make this scale independence explicit, the $\lambda$ dependence of all the couplings (controlled by their $\beta$ functions) has of course also to be taken into account (see Table 1 in Ref. [2] for details).

In Eqs. (9) and (10) $g_{A}^{0}$ denotes the axial coupling constant of the nucleon in the chiral limit. The parameters $F_{\pi}$ and $\Delta_{0}$ are the pion decay constant (with the physical value $92.4 \mathrm{MeV}$ ) and the real part of the $N \Delta$ mass splitting, whereas $c_{A}$ and $g_{1}$ denote the leading axial $N \Delta$ and $\Delta \Delta$ couplings, respectively. It is understood that these four parameters are also taken in the chiral limit.

Throughout this work we have converted the quark-mass dependence of $g_{A}$ into a dependence on the mass of the lowest lying $0^{-}$boson (i.e. the "pion") in the theory via the relation
FIG. 3. Diagrams contributing to the quark-mass dependence of $g_{A}$ up to $\mathcal{O}\left(\epsilon^{3}\right)$ in the SSE scheme. 


$$
m_{\pi}^{2}=2 B_{0} \hat{m}[1+\mathcal{O}(\hat{m})],
$$

where $\hat{m}=\left(m_{u}+m_{d}\right) / 2$ denotes the average of the $u$ and $d$ quark mass and the parameter $B_{0}=-\langle 0|\bar{q} q| 0\rangle / F_{\pi}^{2}$ measures the size of the SU(2) chiral condensate in the chiral limit. The leading term in Eq. (12) corresponds to the GellMann-Oakes-Renner relation [29]. The indicated higher order quark-mass terms only start contributing beyond the $\mathcal{O}\left(\epsilon^{3}\right)$ considered here. We also note that Eq. (12) is consistent with the available lattice QCD data for the quarkmass dependence of the pion mass (see, e.g., Fig. 1 in Ref. [30]).

Here we employ Eq. (9) in a different representation:

$$
\begin{aligned}
g_{A}(\infty)= & g_{A}^{0}+\left[4 B_{9}^{r}(\lambda)-8 g_{A}^{0} B_{20}^{r}(\lambda)-\frac{\left(g_{A}^{0}\right)^{3}}{16 \pi^{2} F_{\pi}^{2}}-\frac{25 c_{A}^{2} g_{1}}{324 \pi^{2} F_{\pi}^{2}}+\frac{19 c_{A}^{2} g_{A}^{0}}{108 \pi^{2} F_{\pi}^{2}}\right] m_{\pi}^{2}-\frac{m_{\pi}^{2}}{4 \pi^{2} F_{\pi}^{2}}\left[\left(g_{A}^{0}\right)^{3}+\frac{1}{2} g_{A}^{0}\right] \ln \frac{m_{\pi}}{\lambda} \\
& +\frac{4 c_{A}^{2} g_{A}^{0}}{27 \pi \Delta_{0} F_{\pi}^{2}} m_{\pi}^{3}+\left[25 c_{A}^{2} g_{1} \Delta_{0}^{2}-57 c_{A}^{2} g_{A}^{0} \Delta_{0}^{2}-24 c_{A}^{2} g_{A}^{0} m_{\pi}^{2}\right] \frac{\sqrt{m_{\pi}^{2}-\Delta_{0}^{2}}}{81 \pi^{2} F_{\pi}^{2} \Delta_{0}} \arccos \frac{\Delta_{0}}{m_{\pi}}+\frac{25 c_{A}^{2} g_{1}\left(2 \Delta_{0}^{2}-m_{\pi}^{2}\right)}{162 \pi^{2} F_{\pi}^{2}} \ln \frac{2 \Delta_{0}}{m_{\pi}} \\
& +\frac{c_{A}^{2} g_{A}^{0}\left(3 m_{\pi}^{2}-38 \Delta_{0}^{2}\right)}{54 \pi^{2} F_{\pi}^{2}} \ln \frac{2 \Delta_{0}}{m_{\pi}}+\mathcal{O}\left(\epsilon^{4}\right) .
\end{aligned}
$$

In Eq. (13) we have analytically continued the logarithms of Eq. (9) to the region $m_{\pi}>\Delta_{0}$, because that is the region where our Monte Carlo data lie. Furthermore we have introduced the couplings $B_{9}^{r}(\lambda)$ and $B_{20}^{r}(\lambda)$ used in heavy baryon chiral perturbation theory (HBChPT), which can be calculated from the SSE couplings via the relations

$$
\begin{aligned}
& B_{9}^{r}(\lambda)^{\mathrm{SSE}}=B_{9}^{r}(\lambda)-\frac{115 c_{A}^{2} g_{1}}{1944 \pi^{2} F_{\pi}^{2}}-\frac{25 c_{A}^{2} g_{1}}{648 \pi^{2} F_{\pi}^{2}} \ln \frac{2 \Delta_{0}}{\lambda}, \\
& B_{20}^{r}(\lambda)^{\mathrm{SSE}}=B_{20}^{r}(\lambda)-\frac{35 c_{A}^{2}}{432 \pi^{2} F_{\pi}^{2}}-\frac{c_{A}^{2}}{144 \pi^{2} F_{\pi}^{2}} \ln \frac{2 \Delta_{0}}{\lambda} .
\end{aligned}
$$

Thus, we are able to exploit directly the empirical information on these couplings [see Eq. (20) below] obtained from a HBChPT calculation of $\pi N \rightarrow \pi \pi N$ in Ref. [31]. Another advantage of utilizing the couplings $B_{9}^{r}(\lambda)$ and $B_{20}^{r}(\lambda)$ becomes clear when we study the chiral limit behavior of Eq. (13):

$$
\begin{aligned}
g_{A}(\infty)= & g_{A}^{0}-\frac{\left(g_{A}^{0}\right)^{3}}{16 \pi^{2} F_{\pi}^{2}} m_{\pi}^{2}+4\left[B_{9}^{r}(\lambda)-2 g_{A}^{0} B_{20}^{r}(\lambda)\right] m_{\pi}^{2} \\
& -\frac{m_{\pi}^{2}}{4 \pi^{2} F_{\pi}^{2}}\left[\left(g_{A}^{0}\right)^{3}+\frac{1}{2} g_{A}^{0}\right] \ln \frac{m_{\pi}}{\lambda}+\mathcal{O}\left(m_{\pi}^{3}\right) .
\end{aligned}
$$

In Eq. (16) it becomes manifest that the couplings defined in Eqs. (14) and (15) ensure that the $\mathcal{O}\left(\epsilon^{3}\right)$ SSE result of Eq. (13) displays the same chiral limit behavior as the $\mathcal{O}\left(p^{3}\right)$ HBChPT result for the quark-mass dependence of $g_{A}$ given in Ref. [32]. In Ref. [3] it was made sure that $g_{A}^{0}$ and the leading nonanalytic quark-mass dependence $\sim \ln m_{\pi}$ agree in the HBChPT and the SSE calculation. Here this mapping of the two field theories is extended to the terms $\sim m_{\pi}^{2}$. A discussion of this point will be given in Ref. [33].

However, we want to emphasize again that the $\mathcal{O}\left(\epsilon^{3}\right)$ SSE results of Refs. [2,3] [i.e. Eq. (9)] and our Eq. (13) are all equivalent and provide identical results, they only differ in the definitions of the employed counterterms.

\section{CHIRAL EFFECTIVE FIELD THEORY (FINITE VOLUME)}

Applying the methods discussed in Ref. [14] (for a recent more detailed investigation of these finite-volume corrections see Ref. [34]), we can now repeat the calculation of $g_{A}$ in the SSE scheme for a finite spatial cubic box of length $L$ in order to obtain the volume dependence of $g_{A}$. We define

$$
g_{A}(L)=g_{A}(\infty)+\Delta g_{A}(L) .
$$

For $g_{A}(\infty)$ we utilize the $\mathcal{O}\left(\epsilon^{3}\right)$ SSE result of Eq. (13). From the diagrams of Fig. 3, we obtain for $\Delta g_{A}(L)$ to $\mathcal{O}\left(\epsilon^{3}\right)$ in SSE

$$
\begin{aligned}
\Delta g_{A}(L)= & -\frac{g_{A}^{0} m_{\pi}^{2}}{4 \pi^{2} F_{\pi}^{2}} \sum_{\vec{n}}^{\prime} \frac{K_{1}\left(L|\vec{n}| m_{\pi}\right)}{L|\vec{n}| m_{\pi}}+\frac{\left(g_{A}^{0}\right)^{3} m_{\pi}^{2}}{6 \pi^{2} F_{\pi}^{2}} \sum_{\vec{n}}^{\prime}\left[K_{0}\left(L|\vec{n}| m_{\pi}\right)-\frac{K_{1}\left(L|\vec{n}| m_{\pi}\right)}{L|\vec{n}| m_{\pi}}\right] \\
& +\frac{25 c_{A}^{2} g_{1}}{81 \pi^{2} F_{\pi}^{2}} \int_{0}^{\infty} d y y \sum_{\vec{n}}^{\prime}\left[K_{0}\left(L|\vec{n}| f\left(m_{\pi}, y\right)\right)-\frac{L|\vec{n}| f\left(m_{\pi}, y\right)}{3} K_{1}\left(L|\vec{n}| f\left(m_{\pi}, y\right)\right)\right] \\
& -\frac{c_{A}^{2} g_{A}^{0}}{\pi^{2} F_{\pi}^{2}} \int_{0}^{\infty} d y y \sum_{\vec{n}}^{\prime}\left[K_{0}\left(L|\vec{n}| f\left(m_{\pi}, y\right)\right)-\frac{L|\vec{n}| f\left(m_{\pi}, y\right)}{3} K_{1}\left(L|\vec{n}| f\left(m_{\pi}, y\right)\right)\right]+\frac{8 c_{A}^{2} g_{A}^{0}}{27 \pi^{2} F_{\pi}^{2}} \int_{0}^{\infty} d y \sum_{\vec{n}}^{\prime} \frac{f\left(m_{\pi}, y\right)^{2}}{\Delta_{0}} \\
& \times\left[K_{0}\left(L|\vec{n}| f\left(m_{\pi}, y\right)\right)-\frac{K_{1}\left(L|\vec{n}| f\left(m_{\pi}, y\right)\right)}{L|\vec{n}| f\left(m_{\pi}, y\right)}\right]-\frac{4 c_{A}^{2} g_{A}^{0}}{27 \pi F_{\pi}^{2}} \frac{m_{\pi}^{3}}{\Delta_{0}} \sum_{\vec{n}}^{\prime} \frac{1}{L|\vec{n}| m_{\pi}} e^{-L|\vec{n}| m_{\pi}}+\mathcal{O}\left(\epsilon^{4}\right)
\end{aligned}
$$


with

$$
f\left(m_{\pi}, y\right)=\sqrt{m_{\pi}^{2}+y^{2}+2 y \Delta_{0}} .
$$

The $\Sigma^{\prime}$ indicates the omission of the $|\vec{n}|=0$ contribution in the sum over all vectors $\vec{n}$ with integer components. The contributions of the individual Feynman diagrams shown in Fig. 3 to $\Delta g_{A}$ are given in the Appendix. The axial coupling of the nucleon in a finite volume depends on the very same parameters as the infinite-volume result.

Note that the recent analysis of Ref. [27] is based on the calculation by Beane and Savage [7], which does not employ decoupling constraints in the chiral extrapolation function of $g_{A}(\infty)$. Presumably this results in a fit function where the coupling $g_{A}^{0}$ does not agree with the value of $g_{A}$ at vanishing pion mass. This in turn could lead to different predictions for the finite-volume corrections, since the analytical formula for $\Delta g_{A}(L)$ should be independent of the use of decoupling constraints but is expressed in terms of the same parameters as the infinite-volume result. In the Appendix we attempt a comparison between our result for $\Delta g_{A}(L)$ of Eq. (18) and the calculation by Beane and Savage [7].

Equation (17) now allows us to access a larger set of lattice data than the formula (13), without introducing new parameters.

\section{FIT RESULTS}

As in previous applications of SSE results to the chiral extrapolation of baryon properties (see, e.g., Refs. [3,28,35]), we do not have enough data points at sufficiently small masses to fit all parameters. So we must fix some of the parameters at reasonable values. What is known from phenomenology? The analysis of (inelastic) $\pi N$ scattering, in particular, the process $\pi N \rightarrow$ $\pi \pi N$ [31], suggests that choosing the physical pion mass as the scale $\lambda$ one has [3]

$$
\begin{aligned}
B_{9}^{r}\left(\lambda=m_{\pi}^{\text {phys }}\right) & =(-1.4 \pm 1.2) \mathrm{GeV}^{-2}, \\
B_{20}^{r}\left(\lambda=m_{\pi}^{\text {phys }}\right) & \equiv 0 .
\end{aligned}
$$

The coupling $B_{20}^{r}$ cannot be observed independently of $B_{9}^{r}$, as it accompanies a structure in the chiral Lagrangian which is proportional to the equation of motion (see e.g. Ref. [36]). Hence, the separation between the two couplings given by Eq. (20) can be utilized without imposing any model assumptions.

Therefore we set $\lambda=0.14 \mathrm{GeV}$ in the following and take $B_{20}^{r}$ to be zero in order to utilize this valuable information from scattering experiments. Furthermore, from analyses of $\pi N$ scattering and $\pi$-photoproduction in the $\Delta$ (1232) resonance region, one knows [37] the real part of the $N \Delta$ mass splitting to be $0.271 \mathrm{GeV}$ at the physical point. We note that a recent analysis [38] of the quark-mass dependence of the (real part of the) mass of $\Delta(1232)$ found a slightly higher value for the splitting between the mass of the nucleon and its first excited state in the chiral limit, $\Delta_{0} \approx 330 \mathrm{MeV}$, albeit with a large uncertainty. We will study the implications of this finding in a future communication [33]. The imaginary part of the complex mass of $\Delta(1232)$ is also known [37]: In an $O\left(\epsilon^{3}\right)$ analysis within SSE it corresponds to a strong decay into $\pi N$ intermediate states with the coupling value $c_{A}=1.5$ [39]. In the previous analysis of the quark-mass dependence of $g_{A}$ of Ref. [3] a smaller value for $c_{A}$ was utilized. Ultimately such an issue can only be decided by performing simultaneous global fits to several observables sensitive to $\Delta(1232)$ contributions within the same ChEFT formalism.

At the physical pion mass we also know the very precise value for the axial coupling $g_{A}^{\exp }=1.2695$ of the nucleon from neutron beta decay analyses [37], which in the $\mathcal{O}\left(\epsilon^{3}\right)$ SSE analysis of Ref. [3] together with a set of quenched lattice data led to the estimate $g_{A}^{0} \approx 1.2$. Little is known about $g_{1}$. In the SU(6) quark model, one would expect $g_{1}=\frac{9}{5} g_{A}^{0} \approx \frac{9}{5} 1.2=2.16$. For the pion decay constant ChPT analyses suggest a chiral limit value of $F_{\pi} \approx$ $86.2 \mathrm{MeV}$ [40]. In the following we fix $\Delta_{0}=$ $0.271 \mathrm{GeV}, c_{A}=1.5, F_{\pi}=86.2 \mathrm{MeV}$, and leave $g_{A}^{0}, g_{1}$, $B_{9}^{r} \equiv B_{9}^{r}(\lambda=0.14 \mathrm{GeV})$ as fit parameters.

To be on the safe side as far as the applicability of ChEFT is concerned, we start with a fit that takes into account only the six data points at our lowest quark masses (data points 3, 6, 12, 13, 14, and 17 in Tables I and II). Indeed, we cannot expect ChEFT to work at pion masses well above 500-600 MeV, because the pion-loop integrals start to become more and more sensitive to scales beyond $\Lambda_{\chi} \sim 4 \pi F_{\pi}$ for such large masses (see the discussion in Ref. [41] for details). Fortunately, we can include one $(\beta, \kappa)$ pair where we have simulations for three different volumes.

Thus, we deal with a three-parameter fit of six data points, which we shall call Fit A. In the fit function we have, of course, to include the finite-volume correction, i.e. we use $g_{A}(L)=g_{A}(\infty)+\Delta g_{A}(L)$ with $g_{A}(\infty)$ given in Eq. (13) and $\Delta g_{A}(L)$ taken from Eq. (18). The resulting values for the fit parameters are given in Table IV.

In Fig. 4 we plot the data with the finite size correction $\Delta g_{A}(L)$ subtracted together with the fit curve. If the fit would describe the volume dependence of the data perfectly, the data points from simulations differing only in the volume (simulations 12,13, and 14) would fall on top of each other in the plot. As can be seen in Fig. 4, this is indeed rather well satisfied within the error bars.

TABLE IV. Fit results.

\begin{tabular}{lllcc}
\hline \hline fit & \multicolumn{1}{c}{$g_{A}^{0}$} & \multicolumn{1}{c}{$B_{9}^{r}$} & $g_{1}$ & $\chi^{2} / \mathrm{dof}$ \\
\hline A & $1.15(12)$ & $-0.71(18) \mathrm{GeV}^{-2}$ & $2.6(8)$ & 1.41 \\
B & $1.26(8)$ & $-0.62(3) \mathrm{GeV}^{-2}$ & $3.3(7)$ & 1.54 \\
\hline \hline
\end{tabular}




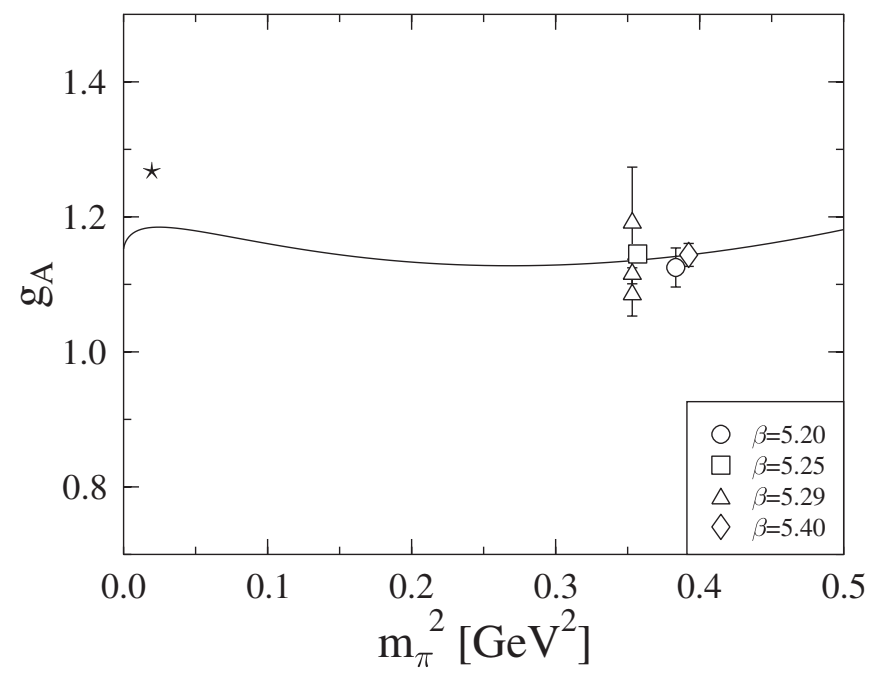

FIG. 4. Fit A of the $g_{A}$ data. The finite size correction has been subtracted from the simulation results. Only data points included in the fit are shown. The star represents the physical point.

Furthermore, subtracting $\Delta g_{A}(L)$ has moved the "large volume" results at our smallest quark masses from all four $\beta$ values even closer together, corroborating our previous impression that lattice artifacts are small.

Remarkably enough, our fits do not break down when data at somewhat higher pion masses are included. Indeed, if we take into account also the data from simulations 9, 10, and 11 , where $m_{\pi} \approx 0.77 \mathrm{GeV}$, we obtain the results labeled as Fit B in Table IV. They are well compatible with the numbers from Fit A. In Fig. 5 the fit curve is confronted with the data.

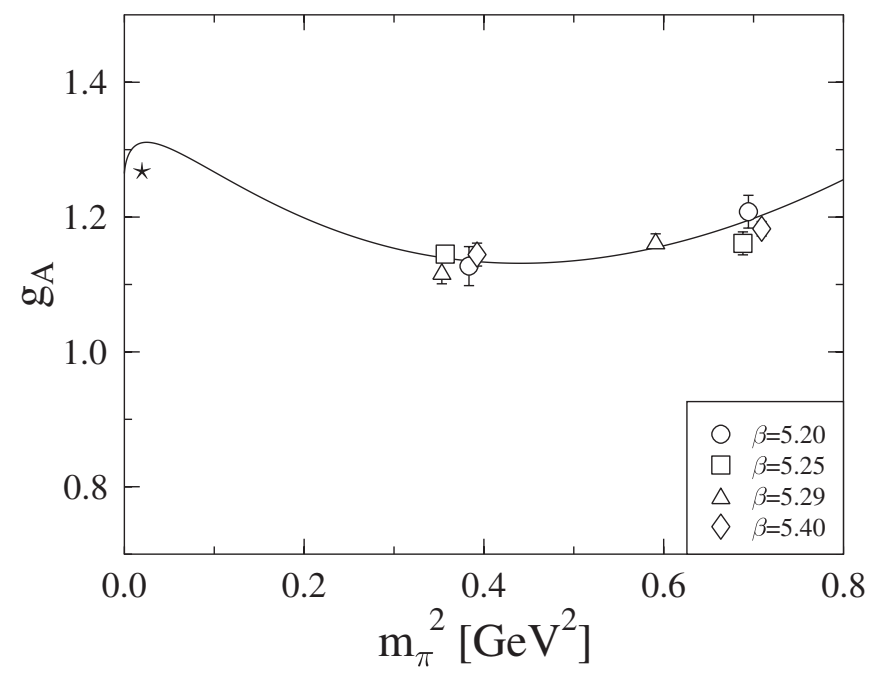

FIG. 5. Fit of the $g_{A}$ data over an extended range of pion masses (Fit B). The finite size correction has been subtracted from the data. In contrast to Fig. 4, only the resulting infinitevolume numbers are shown for the two masses at $\beta=5.29$. The three data points around $m_{\pi}^{2} \approx 0.7 \mathrm{GeV}^{2}$ have not been included in the fit.
Using the fit parameters from Fit B, we plot in Fig. 6 the dependence of $g_{A}$ on the box size $L$ for $m_{\pi}=0.594 \mathrm{GeV}$ together with our three data points at $\beta=5.29, \kappa=$ 0.1359 and for $m_{\pi}=0.769 \mathrm{GeV}$ along with the three data points at $\beta=5.29, \kappa=0.1355$. In addition, we show the behavior to be expected at $m_{\pi}=0.35 \mathrm{GeV}$ and at the physical pion mass. These plots show clearly how well Fit B reproduces the volume dependence of our data.

In Fig. 7 we display the volume dependence of the data and the fit in yet another way. There we plot our results as they were obtained in the respective volumes versus $m_{\pi}^{2}$. For the curves in this plot, we take the parameters from Fit B. The finite $L$ values correspond to the volumes used in the simulations 12,13 , and 14 .
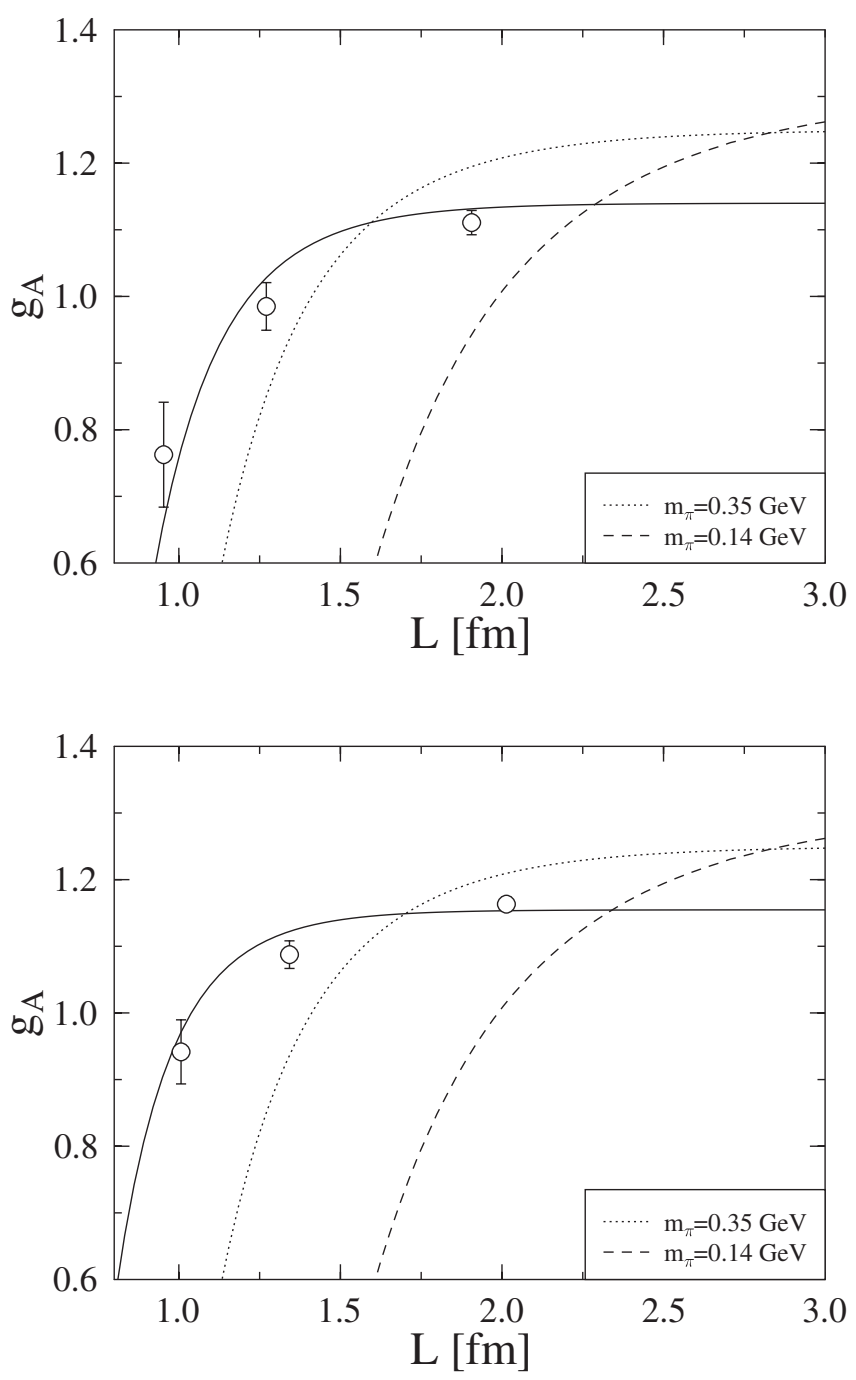

FIG. 6. Results for $g_{A}$ at $\beta=5.29, \kappa=0.1359$ (corresponding to a pion mass of about $0.6 \mathrm{GeV}$, upper plot) and at $\beta=5.29$, $\kappa=0.1355$ (corresponding to a pion mass of about $0.77 \mathrm{GeV}$, lower plot) plotted versus $L$. The full curves have been computed for the corresponding pion masses using the parameters from Fit B. The dotted (dashed) curves show the volume dependence expected from these parameters for $m_{\pi}=0.35 \mathrm{GeV}\left(m_{\pi}=\right.$ $0.14 \mathrm{GeV}$ ). 


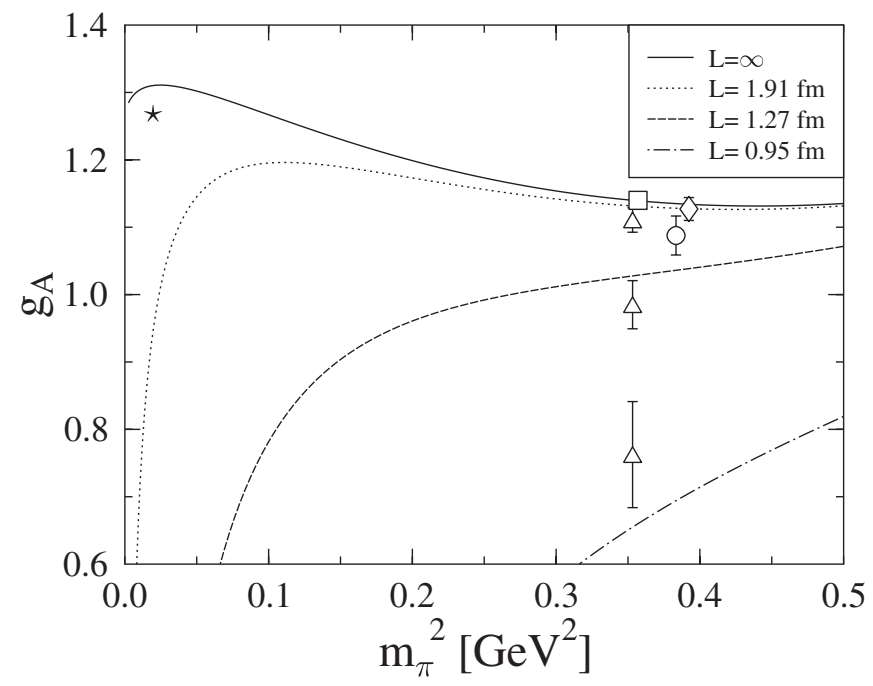

FIG. 7. Results for $g_{A}$ in the simulated volumes together with fit curves from Fit B.

In order to study the influence of the smaller volumes (simulations 9, 10 and 12,13), we have repeated Fit B without these small-volume data, i.e. using only the results from simulations $3,6,11,14$, and 17 . This yields $g_{A}^{0}=$ $1.21 \pm 0.19, \quad B_{9}^{r}=(-0.62 \pm 0.06) \mathrm{GeV}^{-2}, \quad$ and $g_{1}=$ $2.8 \pm 1.7$. Of course, the errors have increased, but the fitted parameters are fully compatible with the outcome of Fit B.

In order to estimate the uncertainty caused by the ambiguities inherent in the scale setting procedure (see Sect. II), we have not only employed $r_{0}=0.467 \mathrm{fm}$ in the fits but also $r_{0}=0.5 \mathrm{fm}$. Moreover, we have considered the two possibilities of working with $r_{0} / a$ evaluated at the respective quark mass and using the chirally extrapolated value of $r_{0} / a$. For $g_{A}^{0}$ we find numbers between 1.12 and 1.21 in Fit A, while they vary between 1.19 and 1.33 in Fit B. The results for $B_{9}^{r}\left(\mathrm{in}_{\mathrm{GeV}}^{-2}\right.$ ) lie between -0.60 and -0.83 in Fit $A$ and between -0.54 and -0.72 in Fit $B$. The values for $g_{1}$ range between 2.1 and 3.2 in Fit A and between 2.5 and 4.1 in Fit B. We shall take half of these spreads as our estimate of the systematic error due to setting the scale.

Let us now discuss the values that our fits have yielded for the parameters. For the axial coupling in the chiral limit $g_{A}^{0}$, we find a result below the value at the physical point. This is in agreement with the analysis of Ref. [3] based on the quenched data of QCDSF [42]. The small negative value for the coupling $B_{9}^{r}$ is also entirely within expectations from the analyses of inelastic $\pi N$-scattering [3,31] [see Eq. (20)]. Finally, we note that the value for the axial $\Delta \Delta$ coupling $g_{1}$ in this new analysis is much closer to the $\mathrm{SU}(6)$ quark model result than the number found in Ref. [3], reassuring us that the contributions of $\Delta(1232)$ to the axial coupling of the nucleon are under control. The main cause for this more reasonable value of $g_{1}$ is the larger value for the coupling $c_{A}$ utilized in the present study [43].
We note that the curves for $g_{A}$ in a finite volume presented previously in Ref. [10] — which were consistently above our infinite-volume curve — can be obtained from the present analysis by setting all $\Delta(1232)$ contributions in $\Delta g_{A}(L)$ identically to zero. The complete finite-volume effects found here within $\mathcal{O}\left(\epsilon^{3}\right)$ SSE, in contrast, are negative at large quark masses, consistent with the findings from the simulation. The resulting finite-volume corrections therefore arise from a destructive interference between $N \pi$ and $\Delta \pi$ loop effects. This point will be discussed in more detail in a future publication [33]. Such a strong cancellation between Goldstone boson loops around octet and decuplet baryon intermediate states has also been observed in Ref. [44] in an SU(3) HBChPT calculation of the axial current of the nucleon in infinite volume, albeit under the additional assumption of degenerate octet-decuplet multiplets.

\section{SUMMARY}

We have evaluated the axial coupling constant $g_{A}$ of the nucleon in lattice QCD on gauge field configurations generated with two degenerate flavors of dynamical nonperturbatively improved Wilson fermions. The necessary renormalization of the axial-vector current has been performed nonperturbatively within the $\mathrm{RI}^{\prime}-\mathrm{MOM}$ scheme, except for the mass-dependent factor: $b_{A}$ has been calculated in tadpole improved boosted perturbation theory.

For two $(\beta, \kappa)$ combinations, we have performed simulations on three different volumes allowing us to study finite size effects. For the chiral extrapolation as well as for the description of the volume dependence, we have made use of ChEFT. For this purpose we have rewritten the expression for the quark-mass dependence of $g_{A}$ derived within the framework of the SSE in Ref. [3] in a form which is particularly suitable for our application, and we have extended the calculation to cover also the volume dependence. With this formula at hand, we could perform a fit to our data obtained on lattices of different spatial extent. Note, however, that we had to fix some parameters at phenomenologically reasonable values. Then finitevolume effects are reproduced surprisingly well down to box lengths of about $1 \mathrm{fm}$. Nevertheless, simulations at smaller quark masses will be necessary to confirm our findings.

In Ref. [45] it has been argued that, in the chiral limit, $1 / 3$ of the axial charge of the nucleon is to be found at infinite distance from the nucleon, due to a delocalization effect. Furthermore, it was suggested that this phenomenon could lead to large finite-volume effects. While there is agreement on the delocalization phenomenon, it has been disputed that this effect will cause calculations of $g_{A}$ in a finite system to have particularly large finite-volume effects [46]. Notwithstanding, our fits suggest, for example, that at the physical pion mass the axial charge is reduced by a factor $\approx 2$ if the nucleon is confined to a periodic box of 
extent $L \approx 1.5 \mathrm{fm}$. This result is in good agreement with a model calculation [9], in which the effect is attributed to periodic boundary conditions rather than delocalization of the axial charge.

Given that the SSE is based upon the long distance pion dynamics around nucleon and $\Delta(1232)$ matter states, we conclude that contributions from $\Delta(1232)$ are crucial in understanding the quark-mass (and volume) dependence of $g_{A}$. This confirms the findings of Ref. [3] and the old expectations (at the physical point) based on the observation that the integral in the Adler-Weisberger sum rule, which represents $g_{A}^{2}-1$, is dominated by the $\Delta(1232)$ resonance. The values for the couplings involving $\Delta(1232)$ dynamics employed in our chiral extrapolation curve are consistent both with scattering phenomenology and with the expectations of the SU(6) quark model. In particular, the value for the axial $\Delta \Delta$ SSE coupling $g_{1} \approx$ 3.0 in this new analysis is much more consistent with expectations from phenomenology than the value obtained in Ref. [3].

Inclusion of simulation data at several values of the box length $L$ is crucial to increase the number of data points at sufficiently small pion masses. The two-dimensional surface $\left(m_{\pi}, L\right)$ allows for a much better determination of the individual values of the effective couplings. With the new dynamical simulation data presented here and our $\mathcal{O}\left(\epsilon^{3}\right)$ SSE analysis, we can extract the chiral limit value $g_{A}^{0}$ from the extrapolation without including any constraints on the physical point. As our final result, we quote $g_{A}^{0}$ from Fit B, which takes into account pion masses below $800 \mathrm{MeV}$. We find

$$
g_{A}^{0}=1.26(8)(7) \text {. }
$$

The first error is statistical, while the second error is an estimate of the systematic uncertainty caused by the ambiguities in setting the scale. Repeating the fit with the value of $g_{A}$ at the physical pion mass as fit parameter instead of $g_{A}^{0}$, we obtain

$$
g_{A}\left(m_{\pi}^{\text {phys }}\right)=1.31(9)(7)
$$

Ultimately, one would like to determine all parameters of ChEFT solely from a fit to lattice data. Such an enterprise would require a joint fit of results from simulations with dynamical quarks for many static nucleon and $\Delta(1232)$ observables, which presently is out of reach due to the paucity of lattice data for pion masses below $600 \mathrm{MeV}$ in many of these observables.

\section{ACKNOWLEDGMENTS}

The numerical calculations have been performed on the Hitachi SR8000 at LRZ (Munich), on the Cray T3E at EPCC (Edinburgh) [47], and on the APEmille at NIC/ DESY (Zeuthen). This work is supported in part by the DFG (Forschergruppe Gitter-Hadronen-Phänomenologie and Emmy-Noether program) and by the EU Integrated Infrastructure Initiative Hadron Physics under Contract No. RII3-CT-2004-506078. A. A. thanks the "Berliner Programm zur Förderung der Chancengleichheit für Frauen in Forschung und Lehre" for financial support. T.R.H. acknowledges the hospitality of the Forschergruppe "Gitter-Hadronen-Phänomenologie" at the University of Regensburg and at DESY Zeuthen.

\section{APPENDIX: AMPLITUDES IN FINITE VOLUME}

The amplitudes of the contributions of the eight Feynman diagrams of Fig. 3 to $g_{A}$ in the infinite volume can be found in the appendix of Ref. [2]. Here we give the individual contributions of the eight diagrams to $\Delta g_{A}$ defined in Eq. (17):

$$
\begin{aligned}
\Delta A m p_{1}= & \mathrm{i}\left(\eta^{\dagger} \tau^{b} \eta\right) \times \bar{u}\left(r_{1}\right) S \cdot \epsilon_{A} u\left(r_{2}\right)\left[\frac{9\left(g_{A}^{0}\right)^{3}}{4 F_{\pi}^{2}}\left(\frac{m_{\pi}^{2}}{12 \pi^{2}} \sum_{\vec{n}}^{\prime} K_{0}\left(L|\vec{n}| m_{\pi}\right)-\frac{m_{\pi}}{12 \pi^{2} L} \sum_{\vec{n}}^{\prime} \frac{1}{|\vec{n}|} K_{1}\left(L|\vec{n}| m_{\pi}\right)\right)\right. \\
& \left.-\frac{c_{A}^{2} g_{A}^{0}}{\pi^{2} F_{\pi}^{2}} \int_{0}^{\infty} \mathrm{d} y y \sum_{\vec{n}}^{\prime}\left(K_{0}\left(L|\vec{n}| \sqrt{m_{\pi}^{2}+y^{2}+2 y \Delta_{0}}\right)-\frac{1}{3} L|\vec{n}| \sqrt{m_{\pi}^{2}+y^{2}+2 y \Delta_{0}} K_{1}\left(L|\vec{n}| \sqrt{m_{\pi}^{2}+y^{2}+2 y \Delta_{0}}\right)\right)\right]
\end{aligned}
$$

$$
\Delta A m p_{2}=\Delta A m p_{3}=0,
$$

$$
\Delta A m p_{4}=-\mathrm{i}\left(\eta^{\dagger} \tau^{b} \eta\right) \times \bar{u}\left(r_{1}\right) S \cdot \epsilon_{A} u\left(r_{2}\right) \frac{\left(g_{A}^{0}\right)^{3}}{4 F_{\pi}^{2}}\left(\frac{m_{\pi}^{2}}{12 \pi^{2}} \sum_{\vec{n}}^{\prime} K_{0}\left(L|\vec{n}| m_{\pi}\right)-\frac{m_{\pi}}{12 \pi^{2} L} \sum_{\vec{n}}^{\prime} \frac{1}{|\vec{n}|} K_{1}\left(L|\vec{n}| m_{\pi}\right)\right),
$$

$$
\Delta A m p_{5}=-\mathrm{i}\left(\eta^{\dagger} \tau^{b} \eta\right) \times \bar{u}\left(r_{1}\right) S \cdot \epsilon_{A} u\left(r_{2}\right) \frac{g_{A}^{0} m_{\pi}}{4 \pi^{2} F_{\pi}^{2} L} \sum_{\vec{n}}^{\prime} \frac{1}{|\vec{n}|} K_{1}\left(L|\vec{n}| m_{\pi}\right)
$$


TABLE V. The correspondence (at leading order) between the SSE couplings used in this work and the parameters used in Ref. [7].

\begin{tabular}{lc}
\hline \hline SSE couplings & Couplings of Ref. [7] \\
\hline$g_{A}^{0}$ & $g_{A}^{\mathrm{BS}}$ \\
$g_{1}$ & $-g_{\Delta \Delta}^{\mathrm{BS}}$ \\
$c_{A}$ & $-\left(g_{\Delta N}^{\mathrm{BS}} / \sqrt{2}\right)$ \\
$\sqrt{2} F_{\pi}$ & $f_{\mathrm{BS}}$ \\
$\Delta_{0}$ & $\Delta_{\mathrm{BS}}$ \\
\hline \hline
\end{tabular}

TABLE VI. The two coupling scenarios discussed in Ref. [7].

\begin{tabular}{lll}
\hline \hline Parameter & \multicolumn{1}{c}{ (a) } & \multicolumn{1}{c}{ (b) } \\
\hline$g_{A}^{\mathrm{BS}}$ & 1.67 & 1.33 \\
$\Delta_{\mathrm{BS}}$ & $0.293 \mathrm{GeV}$ & $0.293 \mathrm{GeV}$ \\
$g_{\Delta N}^{\mathrm{BS}}$ & -2 & -1.41 \\
$g_{\Delta \Delta}^{\mathrm{BS}}$ & -3 & -3 \\
$f_{\mathrm{BS}}$ & $0.132 \mathrm{GeV}$ & $0.132 \mathrm{GeV}$ \\
\hline \hline
\end{tabular}

$$
\begin{aligned}
\Delta A m p_{6}= & \Delta A m p_{7} \\
= & \mathrm{i}\left(\eta^{\dagger} \tau^{b} \eta\right) \times \bar{u}\left(r_{1}\right) S \cdot \epsilon_{A} u\left(r_{2}\right) \frac{4 g_{A}^{0} c_{A}^{2}}{27 \pi^{2} \Delta_{0} F_{\pi}^{2}}\left[\int _ { 0 } ^ { \infty } \mathrm { d } y \sum _ { \vec { n } } ^ { \prime } \left(\sqrt { m _ { \pi } ^ { 2 } + y ^ { 2 } + 2 y \Delta _ { 0 } } \left(-\frac{1}{L|\vec{n}|} K_{1}\left(L|\vec{n}| \sqrt{m_{\pi}^{2}+y^{2}+2 y \Delta_{0}}\right)\right.\right.\right. \\
& \left.\left.+\sqrt{m_{\pi}^{2}+y^{2}+2 y \Delta_{0}} K_{0}\left(L|\vec{n}| \sqrt{m_{\pi}^{2}+y^{2}+2 y \Delta_{0}}\right)\right)-\frac{m_{\pi}^{2} \pi}{2} \sum_{\vec{n}}^{\prime} \frac{1}{L|\vec{n}|} e^{-L|\vec{n}| m_{\pi}}\right], \\
& \quad \Delta A m p_{8}=\mathrm{i}\left(\eta^{\dagger} \tau^{b} \eta\right) \times \bar{u}\left(r_{1}\right) S \cdot \epsilon_{A} u\left(r_{2}\right) \frac{25}{81} \frac{c_{A}^{2} g_{1}}{\pi^{2} F_{\pi}^{2}} \int_{0}^{\infty} \mathrm{d} y y \sum_{\vec{n}}^{\prime}\left(K_{0}\left(L|\vec{n}| \sqrt{m_{\pi}^{2}+y^{2}+2 y \Delta_{0}}\right)\right. \\
& \left.-\frac{1}{3} L|\vec{n}| \sqrt{m_{\pi}^{2}+y^{2}+2 y \Delta_{0}} K_{1}\left(L|\vec{n}| \sqrt{m_{\pi}^{2}+y^{2}+2 y \Delta_{0}}\right)\right) .
\end{aligned}
$$

Additional details are given in Ref. [43]. Note that the finite-volume shift in diagram 1 arises from the $Z$ factor of the nucleon. In Eqs. (A1)-(A6) $S_{\mu}$ denotes the PauliLubanski vector of ChEFT, whereas $\epsilon_{A}^{\mu}$ corresponds to an external axial-vector background source interacting with the hadronic system; $u\left(r_{1}\right), \bar{u}\left(r_{2}\right)$ are the nonrelativistic spinors of the incoming/outgoing nucleon with 4-momenta $r_{1}^{\mu}, r_{2}^{\mu}$, respectively. The bilinear combination $\eta^{\dagger} \tau^{b} \eta$ with $b=1,2,3$ denoting the isovector index of the background source encodes the isospin dependence of the current. The sums extend over all vectors $\vec{n}$ with integer components excluding $\vec{n}=\overrightarrow{0}$. For further details on the notation we refer to Ref. [2]. The couplings and parameters occurring in these relations are defined in the main text.

In Table $\mathrm{V}$ we have attempted to relate our couplings $g_{A}^{0}$, $c_{A}, g_{1}, \Delta_{0}$, and $F_{\pi}$ to the ones used by Beane and Savage in Ref. [7]. However, due to the decoupling constraints of the SSE scheme (see the discussion in Sec. III), the correspondence between the two sets of couplings shown in Table V is strictly true only at leading order in the chiral expansion.
While we are also employing a different representation of the finite-volume shifts in terms of the Bessel functions, numerically we can reproduce the results shown in Ref. [7] by utilizing the two parameter sets discussed there and shown in Table VI. However, the sign of the finite-volume shift observed in the Monte-Carlo simulation of $g_{A}$ reported in this work does not agree with either of these two coupling scenarios, which would both lead to an increase of $g_{A}$ when the volume is decreased for pion masses above $140 \mathrm{MeV}$. Finally, we emphasize again that our set of couplings of the effective theory in the SSE scheme has been obtained for the complete result $g_{A}(L)$ of Eq. (17), and not just for the finite-volume shift $\Delta g_{A}(L)$ as discussed in Ref. [7]. At $\mathcal{O}\left(\epsilon^{3}\right)$, differences between the SSE scheme used in this work and the approach of Ref. [7] - aside from numerical differences in the couplings - only manifest themselves in the $g_{A}(\infty)$ part of $g_{A}(L)$.
[1] T. R. Hemmert, B. R. Holstein, and J. Kambor, J. Phys. G 24, 1831 (1998).

[2] V. Bernard, H.W. Fearing, T. R. Hemmert, and U.-G. Meißner, Nucl. Phys. A635, 121 (1998); A642, 563(E) (1998).
[3] T. R. Hemmert, M. Procura, and W. Weise, Phys. Rev. D 68, 075009 (2003).

[4] S. R. Beane and M. J. Savage, Phys. Rev. D 68, 114502 (2003).

[5] O. Bär, Nucl. Phys. B, Proc. Suppl. 140, 106 (2005). 
[6] W. Detmold, W. Melnitchouk, and A. W. Thomas, Phys. Rev. D 66, 054501 (2002).

[7] S. R. Beane and M.J. Savage, Phys. Rev. D 70, 074029 (2004).

[8] W. Detmold and C. J. Lin, Phys. Rev. D 71, 054510 (2005).

[9] A. W. Thomas, J. D. Ashley, D. B. Leinweber, and R. D. Young, J. Phys.: Conf. Ser. 9, 321 (2005).

[10] A. Ali Khan, M. Göckeler, P. Hägler, T. R. Hemmert, R. Horsley, A. C. Irving, D. Pleiter, P.E. L. Rakow, A. Schäfer, G. Schierholz, H. Stüben, and J. Zanotti (QCDSF-UKQCD Collaboration), Nucl. Phys. B, Proc. Suppl. 140, 408 (2005).

[11] A. Ali Khan, M. Göckeler, Ph. Hägler, T. R. Hemmert, R. Horsley, A. C. Irving, D. Pleiter, P.E. L. Rakow, A. Schäfer, G. Schierholz, H. Stüben, T. Wollenweber, and J.M. Zanotti (QCDSF-UKQCD Collaboration), PoS LAT2005 (2005) 349 [hep-lat/0510061].

[12] R. Sommer, Nucl. Phys. B411, 839 (1994).

[13] M. Lüscher, S. Sint, R. Sommer, and P. Weisz, Nucl. Phys. B478, 365 (1996).

[14] A. Ali Khan, T. Bakeyev, M. Göckeler, T. R. Hemmert, R. Horsley, A. C. Irving, B. Joó, D. Pleiter, P. E. L. Rakow, G. Schierholz, and H. Stüben (QCDSF-UKQCD Collaboration), Nucl. Phys. B689, 175 (2004).

[15] M. Göckeler, R. Horsley, D. Pleiter, P. E. L. Rakow, G. Schierholz, W. Schroers, H. Stüben, and J. M. Zanotti, PoS LAT2005 (2005) 063 [hep-lat/0509196].

[16] C. Aubin, C. Bernard, C. DeTar, S. A. Gottlieb, E. B. Gregory, U. M. Heller, J. E. Hetrick, J. Osborn, R. Sugar, and D. Toussaint, Phys. Rev. D 70, 094505 (2004).

[17] M. Della Morte, R. Hoffmann, F. Knechtli, R. Sommer, and U. Wolff, J. High Energy Phys. 07 (2005) 007.

[18] M. Göckeler, R. Horsley, A. C. Irving, D. Pleiter, P. E. L. Rakow, G. Schierholz, and H. Stüben, Phys. Rev. D 73, 014513 (2006).

[19] S. Sint and P. Weisz, Nucl. Phys. B502, 251 (1997).

[20] T. Bhattacharya, R. Gupta, W. J. Lee, and S. R. Sharpe, Phys. Rev. D 63, 074505 (2001).

[21] G. Martinelli, C. Pittori, C. T. Sachrajda, M. Testa, and A. Vladikas, Nucl. Phys. B445, 81 (1995).

[22] M. Göckeler, R. Horsley, H. Oelrich, H. Perlt, D. Petters, P. E. L. Rakow, A. Schäfer, G. Schierholz, and A. Schiller, Nucl. Phys. B544, 699 (1999).

[23] J. A. Gracey, Nucl. Phys. B662, 247 (2003).

[24] S. Sasaki, K. Orginos, S. Ohta, and T. Blum, Phys. Rev. D 68, 054509 (2003).

[25] D. Dolgov, R. Brower, S. Capitani, P. Dreher, J. W. Negele, A. Pochinsky, D. B. Renner, N. Eicker, Th. Lippert, K. Schilling, R. G. Edwards, and U. M. Heller (LHPC and SESAM Collaborations), Phys. Rev. D 66, 034506 (2002).
[26] S. Ohta and K. Orginos (RBCK Collaboration), Nucl. Phys. B, Proc. Suppl. 129, 296 (2004); 140, 396 (2005).

[27] R. G. Edwards, G. T. Fleming, Ph. Hägler, J. W. Negele, K. Orginos, A. V. Pochinsky, D. B. Renner, D. G. Richards, and W. Schroers (LHPC Collaboration), Phys. Rev. Lett. 96, 052001 (2006).

[28] T. R. Hemmert and W. Weise, Eur. Phys. J. A 15, 487 (2002).

[29] M. Gell-Mann, R. J. Oakes, and B. Renner, Phys. Rev. 175, 2195 (1968).

[30] M. Göckeler, hep-lat/0412013.

[31] N. Fettes, Berichte des Forschungszentrums Jülich No. 3814, FZ Jülich (2000).

[32] V. Bernard, N. Kaiser, and U.-G. Meißner, Int. J. Mod. Phys. E 4, 193 (1995).

[33] QCDSF Collaboration (unpublished).

[34] M. Procura, B. U. Musch, T. Wollenweber, T. R. Hemmert, and W. Weise, Phys. Rev. D 73, 114510 (2006).

[35] M. Göckeler, T. R. Hemmert, R. Horsley, D. Pleiter, P. E. L. Rakow, A. Schäfer, and G. Schierholz (QCDSF Collaboration), Phys. Rev. D 71, 034508 (2005).

[36] G. Ecker, Phys. Lett. B 336, 508 (1994).

[37] S. Eidelman et al. (Particle Data Group), Phys. Lett. B 592, 1 (2004).

[38] V. Bernard, T. R. Hemmert, and U.-G. Meißner, Phys. Lett. B 622, 141 (2005).

[39] T. A. Gail and T.R. Hemmert, Eur. Phys. J. A 28, 91 (2006).

[40] G. Colangelo and S. Dürr, Eur. Phys. J. C 33, 543 (2004).

[41] V. Bernard, T. R. Hemmert, and U.-G. Meißner, Nucl. Phys. A732, 149 (2004).

[42] S. Capitani, M. Göckeler, R. Horsley, H. Perlt, D. Petters, D. Pleiter, P. E. L. Rakow, G. Schierholz, A. Schiller, and P. Stephenson, Nucl. Phys. B, Proc. Suppl. 79, 548 (1999); R. Horsley (QCDSF and UKQCD Collaborations), Nucl. Phys. B, Proc. Suppl. 94, 307 (2001); M. Göckeler, R. Horsley, D. Pleiter, P.E. L. Rakow, A. Schäfer, and G. Schierholz, Nucl. Phys. B, Proc. Suppl. 119, 32 (2003).

[43] T. Wollenweber, Diploma thesis, TU München T-39, 2005.

[44] E. Jenkins and A. V. Manohar, Phys. Lett. B 259, 353 (1991).

[45] R. L. Jaffe, Phys. Lett. B 529, 105 (2002).

[46] T. D. Cohen, Phys. Lett. B 529, 50 (2002).

[47] C. R. Allton, S. P. Booth, K. C. Bowler, J. Garden, A. Hart, D. Hepburn, A.C. Irving, B. Joó, R. D. Kenway, C. M. Maynard, C. McNeile, C. Michael, S. M. Pickles, J.C. Sexton, K. J. Sharkey, Z. Sroczynski, M. Talevi, M. Teper, and H. Wittig (UKQCD Collaboration), Phys. Rev. D 65, 054502 (2002). 